\title{
Association of ADAMTS12 polymorphisms with rheumatoid arthritis
}

\author{
SEONG-SU NAH ${ }^{1}$, SOOJEONG LEE ${ }^{2}$, JAESOON JOO ${ }^{2}$, HYUNG-KEE KIM ${ }^{3}$, DONG-RYUL SOHN ${ }^{3}$, \\ JUN-TACK KWON $^{3}$, KEE-MIN WOO ${ }^{4}$, SEUNG-JAE HONG ${ }^{5}$ and HAK-JAE KIM ${ }^{2}$ \\ ${ }^{1}$ Division of Rheumatology, Department of Internal Medicine; Departments of ${ }^{2}$ Microbiology, ${ }^{3}$ Clinical Pharmacology, \\ and ${ }^{4}$ Biochemistry, College of Medicine, Soonchunhyang University, Cheonan; ${ }^{5}$ Division of Rheumatology, \\ Department of Internal Medicine, School of Medicine, Kyung Hee University, Seoul, Republic of Korea
}

Received December 30, 2011; Accepted April 5, 2012

DOI: $10.3892 / \mathrm{mmr} .2012 .867$

\begin{abstract}
ADAM) with thrombospondin type 1 motif 12 (ADAMTS12) is a degradative enzyme that interacts with the degradable fragments of cartilage oligomeric matrix protein, which is a prominent non-collagenous matrix component in articular cartilage. ADAMTS12 has been observed in the cartilage, synovial fluid and serum of arthritic patients, and may play an important role in the pathogenesis of arthritis. In the present study, we investigated whether genetic polymorphisms of ADAMTS12 are associated with rheumatoid arthritis (RA). To observe the association between ADAMTS12 and RA, we genotyped three single nucleotide polymorphisms (SNPs) (rs1364044, intron C/T; rs10461703, intron C/T; rs25754, missense Thr1495Ile) of ADAMTS12 using a direct sequencing method in 303 RA patients and 495 control subjects. Multiple logistic regression models were performed to analyze the genetic data. SNPStats and SNPAnalyzer Pro programs were used to estimate the odds ratios, 95\% confidence intervals and p-values. Bonferroni's correction $\left(\mathrm{p}^{\mathrm{c}}\right)$ was conducted to obtain a defined result. Of the three SNPs, the genotype frequency of rs10461703 was associated with the development of RA $\left(p^{c}=0.0024\right.$ in the co-dominant model; $\mathrm{p}^{\mathrm{c}}=0.0009$ in the dominant model; $\mathrm{p}^{\mathrm{c}}=0.0006$ in the log-additive model). The allele frequency of rs10461703 also showed a significant difference between RA and controls ( $\left.\mathrm{p}^{\mathrm{c}}<0.0001\right)$. The $\mathrm{C}$ allele frequency of $\mathrm{rs} 10461703$ was lower in the RA group (36.6\%) compared to the control group (45.7\%), whereas the T allele frequency of rs10461703 in the RA group $(63.4 \%)$ was higher compared to that in the control group (54.3\%). The other two SNPs (rs1364044 and rs25754) were not associated with the development of RA.
\end{abstract}

Correspondence to: Professor Hak-Jae Kim, Department of Microbiology, College of Medicine, Soonchunhyang University, 366-1 Ssangyongdong, Cheonan 330-090, Republic of Korea

E-mail: hak3962@sch.ac.kr

Key words: a disintegrin and metalloproteinase with a thrombospondin type 1 motif 12 , rheumatoid arthritis, single nucleotide polymorphism
However, we did not find any association between the three tested SNPs and RA patients according to clinical features, including erythrocyte sedimentation rate, $\mathrm{C}$-reactive protein level, rheumatoid factor (+ and -) and bone erosion (+ and -). Our results suggest that ADAMTS12 may be a susceptibility gene for RA development.

\section{Introduction}

Several members of the a disintegrin and metalloproteinase with thrombospondin motifs (ADAMTS) family are known to have significant functions in arthritis. ADAMTS1, ADAMTS4, ADAMTS5 and ADAMTS8 degrade the cartilage proteoglycan aggrecan, and they play a key role in aggrecan loss in arthritis (1-6). Cartilage oligomeric matrix protein (COMP) is a prominent non-collagenous component of cartilage. It accounts for approximately $1 \%$ of the wet weight of articular tissue $(7,8)$. COMP fragments are detected in the cartilage, synovial fluid and serum of osteoarthritis (OA) and rheumatoid arthritis (RA) patients (9). Månsson et al suggested that COMP levels may be used to assess the presence and progression of arthritis (10). ADAMTS12 is a new COMP-degrading enzyme that may play an important role in COMP degradation in the initiation and progression of arthritis (11). ADAMTS12 is also expressed at higher levels in tissues of OA and RA patients $(11,12)$. The ADAMTS12 gene is located on chromosome $5 \mathrm{q} 35$ and spans approximately $365 \mathrm{~kb}$. The chromosome $5 \mathrm{q}$ region has been suggested to contain susceptibility loci for autoimmune or inflammatory diseases, such as Crohn's disease, asthma and RA (13-18). Despite the potential importance of ADAMTS12 on RA pathogenesis, there have been no published studies on the relationship between the genetic variants of ADAMTS12 and RA. Our aim was to investigate whether single nucleotide polymorphisms (SNPs) of ADAMTS12 are associated with RA in the Korean population.

\section{Materials and methods}

Subjects. A case-control study was conducted to determine the genetic association between ADAMTS12 SNPs and RA. Three hundred and three unrelated patients with RA were enrolled from two rheumatic centers (Soonchunhyang 
University Hospital and Kyung Hee University Hospital). Each patient was diagnosed by a rheumatologist according to the ACR 1987 Rheumatoid Arthritis diagnostic criteria (19). Four hundred and ninety-five control subjects were recruited among participants who participated in a general health check-up program. Participants with RA, OA and other severe diseases were excluded. This study was carried out according to the Declaration of Helsinki guidelines, and written informed consent was obtained from each subject. The study was approved by the ethics review committee of the Medical Research Institute, School of Medicine, Kyung Hee University, Seoul, Republic of Korea.

Demographic data were obtained from the patient medical records or by interviews at the time of enrollment. Biochemical data were measured, including erythrocyte sedimentation rate (ESR), C-reactive protein (CRP) and rheumatoid factor (RF). Patients with bone erosion were classified by radiographic findings.

SNP genotyping. We searched ADAMTS12 SNPs using the NCBI websites (www.ensembl.org, www.ncbi.nlm.nih. gov/SNP and www.hapmap.org). SNPs with $>5 \%$ minor allele frequency (MAF), $>10 \%$ heterozygosity and genotype frequencies in the Asian population were included. Among four exonic ADAMTS12 SNPs with $>5 \%$ MAF and $>10 \%$ heterozygosity, a missense SNP rs16891281 (Ser1591Pro) shows a monomorphic genotype in Asians and a synonymous SNP rs61748199 (Leu499Leu) represents no genotypic data in Asians. Therefore, two missense SNPs (rs3813474, Trp1177Arg; rs25754, Thr1495Ile) were selected. Of the intronic SNPs, two SNPs (rs1364044 and rs10461703) were added using the aggressive tagging option of the Tagger program (www.broad. mit.edu/mpg/tagger). Finally, we assessed four SNPs in this study. DNA was isolated from peripheral blood using the GenEx $^{\mathrm{TM}}$ B DNA purification kit (GeneAll Biotechnology, Seoul, Korea). Genomic DNA was amplified by polymerase chain reaction (PCR) using primers for each SNP: rs3813474 (sense, 5'-GGA GGC CTT GTA GCT ACA ACA AC-3' and anti-sense, 5'-GGA AGT TTC AGG TGG TTA CGG TT-3'); rs25754 (sense, 5'-GGA TTG GAG GTG TTC TAC TGG TGT-3' and anti-sense: 5'-TCC ATG TTG GAT GAC ATC AGT GTG-3'); rs1364044 (sense, 5'-TTC CAA ATC CTC CCA TTG TTA CTG CC-3' and anti-sense, 5'-GGA TTG CAA AGG CGA GAT GTG ATG TG-3') and rs10461703 (sense, 5'-TTG GTT TTC CAC CTG GCA TGT GTG TG-3' and anti-sense, 5'-GAG AAT ATG TCC TCA CAG CTT GCC AC-3'). The PCR products were genotyped using the direct sequencing method employing an ABI Prism 3730XL automatic sequencer (PE Applied Biosystems, Foster City, CA, USA). The sequence data was analyzed using SeqManII software (DNAStar Inc., Madison, WI, USA).

Statistical analysis. Hardy-Weinberg equilibrium (HWE) was assessed by SNPStats (http://bioinfo.iconcologia.net/ index.php). Haploview version 4.2 was used for the linkage disequilibrium (LD) block (Daly Lab Inc., Cambridge, MA, USA). SNPStats and SNPAnalyzer Pro (Istech Inc., Goyang, Korea) were also used to evaluate the odds ratios (ORs), 95\% confidence intervals (CIs) and p-values. Multiple logistic regression analysis adjusted for age and gender as covariables
Table I. Clinical and demographic features of the RA and control subjects.

\begin{tabular}{lcc}
\hline & $\begin{array}{c}\text { RA } \\
(\mathrm{n}=303)\end{array}$ & $\begin{array}{c}\text { Control } \\
(\mathrm{n}=495)\end{array}$ \\
\hline Age (years, mean $\pm \mathrm{SD})$ & $49.75 \pm 12.96$ & $43.66 \pm 13.01$ \\
Gender (male/female) & $58 / 245$ & $213 / 282$ \\
Disease duration & $4.66 \pm 4.45$ & \\
(years, mean $\pm \mathrm{SD})$ & & \\
ESR $(\mathrm{mm} / \mathrm{h}$, mean $\pm \mathrm{SD})$ & $41.78 \pm 29.67$ & \\
CRP $(\mathrm{mg} / \mathrm{dl}$, mean $\pm \mathrm{SD})$ & $2.27 \pm 4.86$ & \\
Subgroups & & \\
ESR $(\geq 30 /<30 \mathrm{~mm} / \mathrm{h})$ & $184 / 119$ & \\
CRP $(\geq 0.5 /<0.5 \mathrm{mg} / \mathrm{dl})$ & $210 / 93$ & \\
RF $(+/-)$ & $265 / 38$ & \\
Bone erosion $(+/-)$ & $142 / 161$ & \\
\end{tabular}

RA, rheumatoid arthritis; SD, standard deviation; ESR, erythrocyte sedimentation rate; CRP, C-reactive protein; RF, rheumatoid factor. RA patients with inappropriate clinical data were excluded.

was performed. Bonferroni's correction was applied by multiplying the p-values by the number of SNPs and haplotypes.

\section{Results}

Clinical and demographic features of the study subjects. Table I presents the clinical and demographic features of the RA and control subjects. The mean ages $( \pm \mathrm{SD})$ of RA patients and control subjects were $49.75( \pm 12.96)$ and $43.66( \pm 13.01)$ years, respectively. There were 58 male and 245 female (total $n=303$ ) RA patients and 213 male and 282 female (total $n=495$ ) control subjects. RA patients were classified into clinical subgroups according to ESR levels ( $>30$ and $<30 \mathrm{~mm} / \mathrm{h}$ ), CRP levels $(>0.5$ and $<0.5 \mathrm{mg} / \mathrm{dl}$ ), rheumatoid factor (RF; present and absent) and bone erosion (present and absent). The numbers of RA patients with ESR level $>30$ and $<30 \mathrm{~mm} / \mathrm{h}$ were 184 (60.7\%) and 119 (39.3\%), respectively. The patients with a CRP value $>0.5$ and $<0.5 \mathrm{mg} / \mathrm{dl}$ were $210(69.3 \%)$ and $93(30.7 \%)$, respectively. The patients with a RF present and absent were $265(87.5 \%)$ and $38(12.5 \%)$, respectively. The patients with a bone erosion present and absent were $142(46.9 \%)$ and 161 (53.1\%), respectively.

Genetic association of ADAMTS12 SNPs in RA. The genotype distributions of three SNPs (rs1364044, rs10461703 and rs25754) was within HWE ( $>0.05)$, whereas one SNP (rs3813474) was not $(\mathrm{p}<0.05$; data not shown). Therefore, rs3813474 was excluded in the analysis of genetic data. As a result, the genotypic frequency of rs10461703 was statistically associated with RA in the codominant model [OR=1.70 (2.14), 95\% CI 1.21-2.40 (1.36-3.37), $\left.\mathrm{p}=0.0008, \mathrm{p}^{\mathrm{c}}=0.0024\right]$, the dominant model $\left(\mathrm{OR}=1.82,95 \%\right.$ CI 1.32-2.50, $\left.\mathrm{p}=0.0003, \mathrm{p}^{\mathrm{c}}=0.0009\right)$ and the log-additive model $(\mathrm{OR}=1.51,95 \%$ CI $1.21-1.88$, $\left.\mathrm{p}=0.0002, \mathrm{p}^{\mathrm{c}}=0.0006\right)$. The allele frequency of rs10461703 was also associated with RA $(\mathrm{OR}=0.69,95 \%$ CI $0.56-0.85$, 
Table II. Genotype and allele frequencies of ADAMTS12 SNPs in RA and control subjects.

\begin{tabular}{|c|c|c|c|c|c|c|c|c|c|c|c|}
\hline \multirow[t]{2}{*}{ SNP } & \multirow{2}{*}{$\begin{array}{l}\text { Genotype/ } \\
\text { allele }\end{array}$} & \multicolumn{2}{|c|}{ RA } & \multicolumn{2}{|c|}{ Control } & \multirow[t]{2}{*}{ Model } & \multirow[t]{2}{*}{ OR } & \multicolumn{2}{|c|}{$95 \% \mathrm{CI}$} & \multirow[t]{2}{*}{ p-value } & \multirow[t]{2}{*}{$\mathrm{p}^{\mathrm{c}}$} \\
\hline & & Freq. & $\%$ & Freq. & $\%$ & & & LCL & UCL & & \\
\hline \multirow[t]{8}{*}{ rs1364044 } & $\mathrm{C} / \mathrm{C}$ & 98 & 33.2 & 187 & 39.8 & Co-dominant 1 & 0.80 & 0.57 & 1.14 & 0.3900 & 1.0000 \\
\hline & $\mathrm{C} / \mathrm{T}$ & 141 & 47.8 & 204 & 43.4 & Co-dominant 2 & 0.78 & 0.50 & 1.23 & & \\
\hline & $\mathrm{T} / \mathrm{T}$ & 56 & 19.0 & 79 & 16.8 & Dominant & 0.80 & 0.58 & 1.11 & 0.1700 & 0.5100 \\
\hline & & & & & & Recessive & 0.88 & 0.59 & 1.32 & 0.5400 & 1.0000 \\
\hline & & & & & & Over-dominant & 0.87 & 0.64 & 1.19 & 0.4000 & 1.0000 \\
\hline & & & & & & Log-additive & 0.87 & 0.70 & 1.08 & 0.2100 & 0.6300 \\
\hline & $\mathrm{C}$ & 337 & 57.1 & 578 & 61.5 & & & & & & \\
\hline & $\mathrm{T}$ & 253 & 42.9 & 362 & 38.5 & & 1.20 & 0.97 & 1.48 & 0.0900 & 0.2700 \\
\hline \multirow[t]{8}{*}{ rs10461703 } & $\mathrm{T} / \mathrm{T}$ & 122 & 41.5 & 145 & 29.3 & Co-dominant 1 & 1.70 & 1.21 & 2.40 & 0.0008 & 0.0024 \\
\hline & $\mathrm{T} / \mathrm{C}$ & 129 & 43.9 & 248 & 50.1 & Co-dominant 2 & 2.14 & 1.36 & 3.37 & & \\
\hline & $\mathrm{C} / \mathrm{C}$ & 43 & 14.6 & 102 & 20.6 & Dominant & 1.82 & 1.32 & 2.50 & 0.0003 & 0.0009 \\
\hline & & & & & & Recessive & 1.57 & 1.05 & 2.37 & 0.0270 & 0.0800 \\
\hline & & & & & & Over-dominant & 1.32 & 0.97 & 1.79 & 0.0750 & 0.2300 \\
\hline & & & & & & Log-additive & 1.51 & 1.21 & 1.88 & 0.0002 & 0.0006 \\
\hline & $\mathrm{T}$ & 373 & 63.4 & 538 & 54.3 & & & & & & \\
\hline & $\mathrm{C}$ & 215 & 36.6 & 452 & 45.7 & & 0.69 & 0.56 & 0.85 & $<0.0001$ & $<0.0001$ \\
\hline \multirow[t]{8}{*}{ rs 25754} & $\mathrm{~T} / \mathrm{T}$ & 171 & 58.0 & 272 & 58.2 & Co-dominant 1 & 1.06 & 0.76 & 1.47 & 0.8600 & 1.0000 \\
\hline & $\mathrm{T} / \mathrm{C}$ & 106 & 35.9 & 166 & 35.5 & Co-dominant 2 & 0.88 & 0.46 & 1.70 & & \\
\hline & $\mathrm{C} / \mathrm{C}$ & 18 & 6.1 & 29 & 6.2 & Dominant & 1.03 & 0.75 & 1.41 & 0.8500 & 1.0000 \\
\hline & & 8 & & & & Recessive & 0.86 & 0.45 & 1.64 & 0.6600 & 1.0000 \\
\hline & & & & & & Over-dominant & 1.07 & 0.77 & 1.48 & 0.6800 & 1.0000 \\
\hline & & & & & & Log-additive & 1.00 & 0.77 & 1.29 & 0.9800 & 1.0000 \\
\hline & $\mathrm{T}$ & 448 & 75.9 & 710 & 76.0 & & & & & & \\
\hline & $\mathrm{C}$ & 142 & 24.1 & 224 & 24.0 & & 1.01 & 0.79 & 1.23 & 0.9700 & 1.0000 \\
\hline
\end{tabular}

SNP, single nucleotide polymorphism; RA, rheumatoid arthritis; Freq., frequency; OR, odds ratio; CI, confidence intervals; LCL, lower confidence limit; UCL, upper confidence limit; $\mathrm{p}^{\mathrm{c}}$, p-value corrected by the Bonferroni's method. Total numbers of genotypes and alleles in each SNP are different, since the unclear or missing genotype data were excluded.

Table III. Genotype frequencies of ADAMTS12 SNPs in each population.

\begin{tabular}{lcccccc}
\hline SNP & Genotype & Korean & European & Chinese & Japanese & Sub-Saharan African \\
\hline rs1364044 & C/C & 0.398 & 0.517 & 0.489 & 0.386 & 0.400 \\
& $\mathrm{C} / \mathrm{T}$ & 0.434 & 0.383 & 0.422 & 0.455 & 0.500 \\
& $\mathrm{~T} / \mathrm{T}$ & 0.168 & 0.100 & 0.089 & 0.159 & 0.100 \\
rs10461703 & $\mathrm{T} / \mathrm{T}$ & 0.293 & 0.217 & 0.200 & 0.156 & 0.650 \\
& $\mathrm{~T} / \mathrm{C}$ & 0.501 & 0.550 & 0.556 & 0.578 & 0.267 \\
& $\mathrm{C} / \mathrm{C}$ & 0.206 & 0.233 & 0.244 & 0.267 & 0.083 \\
$\mathrm{rs} 25754$ & $\mathrm{~T} / \mathrm{T}$ & 0.582 & 0.233 & 0.578 & 0.556 & 0.467 \\
& $\mathrm{~T} / \mathrm{A}$ & 0.355 & 0.500 & 0.378 & 0.422 & 0.400 \\
& $\mathrm{~A} / \mathrm{A}$ & 0.062 & 0.267 & 0.044 & 0.022 & 0.133 \\
\hline
\end{tabular}

SNP, single nucleotide polymorphism.

$\left.\mathrm{p}<0.0001, \mathrm{p}^{\mathrm{c}}<0.0001\right)$. The $\mathrm{C}$ allele frequency of rs10461703 was lower in the RA group (36.6\%) than in the control group (45.7\%). The other two SNPs (rs1364044 and rs25754) were not associated with the development of RA (Table II).
Next, we accessed the relationship between the three examined SNPs and clinical features of RA patients, including ESR, CRP, RF (+ and -) and bone erosion (+ and -). However, no significant differences were found in these markers (data 
not shown). The human SNP database (www.ncbi.nlm.nih. gov/SNP, dbSNP Build 132) has the genotype frequencies for rs25754 (T/T:T/A:A/A, European 0.233:0.500:0.267; Chinese 0.578:0.378:0.044; Japanese 0.556:0.422:0.022; Sub-Saharan African 0.467:0.400:0.133), rs10461703 (T/T:T/C:C/C, European 0.217:0.550:0.233; Chinese 0.200:0.556:0.244; Japanese 0.156:0.578:0.267; Sub-Saharan African 0.650:0.267:0.083) and rs1364044 (C/C:C/T:T/T, European 0.517:0.383:0.100; Chinese 0.489:0.422:0.089; Japanese 0.386:0.455:0.159; Sub-Saharan African 0.400:0.500:0.100) (Table III). The genotype distributions of rs1364004 in the control group that were analyzed in our study are similar to those of the Asian population, especially the Japanese population. With respect to rs10461703, the frequencies were similar to those of Europeans, but differences between the populations were not shown. The frequencies of rs25754 in the control group were similar to the Chinese population.

Haplotype association of ADAMTS12 SNPs in Koreans with $R A$. In order to evaluate the haplotype of the polymorphisms within the ADAMTS12 gene, the haplotypes were analyzed by Haploview, SNPanalyzer and SNPstats. A LD block including two SNPs (rs25754 and rs3813474) was constructed using the Gabriel method. However, the haplotype was not analyzed for the association study since a SNP (rs3813474) of the block was not in HWE $(\mathrm{p}<0.05)$.

Sample power. The power of the sample size was calculated using a genetic power calculator (http://pngu.mgh.harvard. edu/ purcell/gpc). When we estimated the sample power ( $\alpha=0.05$, risk 2 -fold, no. of case $80 \%$ power), our case-control study was sufficiently powerful to determine a positive association. In this study, we had 0.9896 for $r s 1364044(n=130)$, 0.9169 for rs $10461703(n=212)$ and 0.9896 for rs25754 $(n=130)$.

\section{Discussion}

Loss of articular cartilage caused by extracellular matrix breakdown is the hallmark of arthritis. Degradative fragments of COMP have been observed in arthritic patients (1). ADAMTS12, a member of the ADAMTS family, has been associated with COMP degradation in vitro, and is significantly overexpressed in the cartilage and synovium of patients with RA (7). Recent studies have demonstrated the importance of COMP degradation by ADAMTS12. In particular, the size of COMP fragments generated by ADAMTS12 is similar to that of COMP-degradative fragments seen in arthritic patients (20). In addition, antibodies against ADAMTS12 significantly inhibit tumor necrosis factor-induced and interleukin-1 $\beta$ induced COMP degradation in cartilage explants. Furthermore, suppression of ADAMTS12 expression using the RNAi method in human chondrocytes markedly prevents COMP degradation (21). COMP degradation mediated by ADAMTS7 and ADAMTS12 is inhibited by $\alpha-2$-macroglobulin (22). The chromosomal location of the ADAMTS12 gene has been suggested to contain susceptibility loci for autoimmune or inflammatory diseases, such as Crohn's disease, asthma and RA (13-18).

A few studies have been published on the role of ADAMTS12 SNPs. Morrison et al reported that an intronic SNP rs6868223 of ADAMTS12 was associated with mortality of heart failure patients of African descent (23). Sun et al showed that the rs2548035 intronic SNP was related to aggressive prostate cancer in a Swedish population (24). Li et al revealed that the rs1423300 intronic SNP was strongly associated with an adverse drug response to gemcitabine (25).

Despite the fact that the ADAMTS12 gene has received attention as being involved in autoimmune diseases, such as RA, the relationship between RA and ADAMTS12 SNPs remains obscure. The results in this study suggest that ADAMTS12 gene variations may be involved in the pathogenesis of RA. In agreement with previous reports, an intronic SNP rs10461703 showed significant association with alleles and genotypes with RA after Bonferroni's correction (corrected $\mathrm{p}=0.0024$ in the codominant model, corrected $\mathrm{p}=0.0015$ in the dominant model, corrected $\mathrm{p}=0.001$ in the $\log$ additive model, corrected $\mathrm{p}<0.0001)$. These facts indicate that there is a potential genetic association between the ADAMTS12 gene and RA. In this study, we selected two missense SNPs (rs3813474, Trp1177Arg; rs25754, Thr1495Ile) for the association study. Of the SNPs, rs 25754 is located in the mRNA sequence of thrombospondin type 1 (8th domain) of ADMTS12. The polymorphism induces a change from threonine at 1475 of the amino acid sequence to isoleucine. The change may or may not result in structural changes of the ADAMTS12 protein and thus may induce different functions of the protein. Moreover, rs3813474 is located in the spacer 2 region of the ADMTS12 gene. The polymorphism induces a change from tryptophan at 1157 of the amino acid sequence to arginine. The SNPs were not associated with RA and its biological and/or clinical significance still need to be elucidated. To confirm the genotype frequencies and the association between the ADAMTS12 gene and RA, replication studies with an adequate sample size or an association study with different SNPs that were not analyzed in this study may be required. Further genetic or biological studies also will help in understanding the precise mechanism of pathogenesis in patients with RA.

In conclusion, we detected a significant association between ADAMTS12 SNPs and rheumatoid arthritis. These findings suggest that the ADAMTS12 genotype may modify the clinical presentation of RA, and this represents another step toward a molecular classification of the genetics of RA.

\section{References}

1. Abbaszade I, Liu RQ, Yang F, et al: Cloning and characterization of ADAMTS11, an aggrecanase from the ADAMTS family. J Biol Chem 274: 23443-23450, 1999.

2. Collins-Racie LA, Flannery CR, Zeng W, Corcoran C, Annis-Freeman B, Agostino MJ, Arai M, DiBlasio-Smith E, Dorner AJ, Georgiadis KE, et al: ADAMTS-8 exhibits aggrecanase activity and is expressed in human articular cartilage. Matrix Biol 23: 219-230, 2004.

3. Kuno K, Okada Y, Kawashima H, Nakamura H, Miyasaka M, Ohno $\mathrm{H}$ and Matsushima K: ADAMTS-1 cleaves a cartilage proteoglycan, aggrecan. FEBS Lett 478: 241-245, 2000.

4. Kuno K, Bannai K, Hakozaki M, Matsushima K and Hirose K: The carboxyl-terminal half region of ADAMTS-1 suppresses both tumorigenicity and experimental tumor metastatic potential. Biochem Biophys Res Commun 319: 1327-1333, 2004

5. Tortorella MD, Burn TC, Pratta MA, et al: Purification and cloning of aggrecanase-1: a member of the ADAMTS family of proteins. Science 284: 1664-1666, 1999. 
6. Tortorella MD, Pratta M, Liu RQ, Austin J, Ross OH, Abbaszade I, Burn T and Arner E: Sites of aggrecan cleavage by recombinant human aggrecanase-1 (ADAMTS-4). J Biol Chem 275: 18566-18573, 2000.

7. DiCesare P, Hauser N, Lehman D, Pasumarti S and Paulsson M Cartilage oligomeric matrix protein (COMP) is an abundant component of tendon. FEBS Lett 354: 237-240, 1994.

8. Hedbom E, Antonsson P, Hjerpe A, Aeschlimann D, Paulsson M, Rosa-Pimentel E, Sommarin Y, Wendel M, Oldberg A and Heinegård D: Cartilage matrix proteins. An acidic oligomeric protein (COMP) detected only in cartilage. J Biol Chem 267: 6132-6136, 1992

9. Di Cesare PE, Carlson CS, Stolerman ES, Hauser N, Tulli H and Paulsson M: Increased degradation and altered tissue distribution of cartilage oligomeric matrix protein in human rheumatoid and osteoarthritic cartilage. J Orthop Res 14: 946-955, 1996.

10. Månsson B, Carey D, Alini M, Ionescu M, Rosenberg LC, Poole AR, Heinegård D and Saxne T: Cartilage and bone metabolism in rheumatoid arthritis. Differences between rapid and slow progression of disease identified by serum markers of cartilage metabolism. J Clin Invest 95: 1071-1077, 1995.

11. Liu CJ, Kong W, Xu K, Luan Y, Ilalov K, Sehgal B, Yu S, Howell RD and Di Cesare PE: ADAMTS-12 associates with and degrades cartilage oligomeric matrix protein. J Biol Chem 281: 15800-15808, 2006

12. Kevorkian L, Young DA, Darrah C, Donell ST, Shepstone L, Porter S, Brockban k SM, Edwards DR, Parker AE and Clark IM: Expression profiling of metalloproteinases and their inhibitors in cartilage. Arthritis Rheum 50: 131-141, 2004

13. Grunig G, Warnock M, Wakil AE, Venkayya R, Brombacher F, Rennick DM, Sheppard D, Mohrs M, Donaldson DD, Locksley RM and Corry DB: Requirement for IL-13 independently of IL-4 in experimental asthma. Science 282: 2261-2263, 1998.

14. Kauppi P, Lindblad-Toh K, Sevon P, Toivonen HT, Rioux JD, Villapakkam A, Laitinen LA, Hudson TJ, Kere J and Laitinen T: A second-generation association study of the $5 \mathrm{q} 31$ cytokine gene cluster and the interleukin- 4 receptor in asthma. Genomics 77 $35-42,2001$

15. Rioux JD, Daly MJ, Silverberg MS, et al: Genetic variation in the $5 \mathrm{q} 31$ cytokine gene cluster confers susceptibility to Crohn disease. Nat Genet 29: 223-228, 2001.
16. Tokuhiro S, Yamada R, Chang X, et al: An intronic SNP in a RUNX1 binding site of SLC22A4, encoding an organic cation transporter, is associated with rheumatoid arthritis. Nat Genet 35: 341-348, 2003

17. Peltekova VD, Wintle RF, Rubin LA, Amos CI, Huang Q, Gu X, Newman B, Van Oene M, Cescon D, Greenberg G, Griffiths AM, St George-Hyslop PH and Siminovitch KA: Functional variants of OCTN cation transporter genes are associated with Crohn disease. Nat Genet 36: 471-475, 2004.

18. Friberg C, Björck K, Nilsson S, Inerot A, Wahlström J and Samuelsson L: Analysis of chromosome 5q31-32 and psoriasis: confirmation of a susceptibility locus but no association with SNPs within SLC22A4 and SLC22A5. J Invest Dermatol 126: 998-1002, 2006.

19. Arnett FC, Edworthy SM, Bloch DA, et al: The American Rheumatism Association 1987 revised criteria for the classification of rheumatoid arthritis. Arthritis Rheum 31: 315-324, 1988.

20. Liu CJ: The role of ADAMTS-7 and ADAMTS-12 in the pathogenesis of arthritis. Nat Clin Pract Rheumatol 5: 38-45, 2009.

21. Bai XH, Wang DW, Luan Y, Yu XP and Liu CJ: Regulation of chondrocyte differentiation by ADAMTS-12 metalloproteinase depends on its enzymatic activity. Cell Mol Life Sci 66: 667-680, 2009.

22. Luan Y, Kong L, Howell DR, Ilalov K, Fajardo M, Bai XH, Di Cesare PE, Goldring MB, Abramson SB and Liu CJ: Inhibition of ADAMTS-7 and ADAMTS-12 degradation of cartilage oligomeric matrix protein by alpha-2-macroglobulin. Osteoarthritis Cartilage 16: 1413-1420, 2008.

23. Morrison AC, Felix JF, Cupples LA, et al: Genomic variation associated with mortality among adults of European and African ancestry with heart failure: the cohorts for heart and aging research in genomic epidemiology consortium. Circ Cardiovasc Genet 3: 248-255, 2010.

24. Sun J,Zheng SL, Wiklund F, et al: Sequence variants at 22q13 are associated with prostate cancer risk. Cancer Res 69: 10-15, 2009.

25. Li L, Fridley BL, Kalari K, Jenkins G, Batzler A, Weinshilboum RM and Wang L: Gemcitabine and arabinosylcytosin pharmacogenomics: genome-wide association and drug response biomarkers. PLoS One 4: e7765, 2009. 